\title{
Well-being and mental health - diet, supplements, exercise or sleep? A review of reports from the last five years
}

Authors' Contribution: A Study Design B Data Collection C Statistical Analysis D Data Interpretation E Manuscript Preparation F Literature Search G Funds Collection

\author{
Elżbieta Grzywacz ${ }^{1}$ ABDEF, Aleksandra Jaroń2 ABDEF \\ ${ }^{1}$ Students scientific club of Oral Surgery in Oral Surgery Department, \\ Pomeranian Medical University in Szczecin, Poland \\ ${ }^{2}$ Department of Oral Surgery, Pomeranian Medical University in Szczecin, Poland
}

\section{abstract}

In the 2020s we are faced with a problem of an ageing society. Not only do we see it and struggle to provide care for the elderly, but we also focus on constantly maintaining a good form related to our well-being as well as physical, mental and social functioning.

The publications used in this review were sourced from the PubMed database and from Google Scholar. The paper uses articles published in English which are not older than 5 years.

Analysis of the latest literature has shown that there is evidence that modifiable factors have a positive effect on our well-being and health.

The best effect can be achieved by combining positive actions in the field of diet, supplementation, moderate but systematic physical activity, and adding the right amount of sleep per day.

Key words: mental health, diet, supplementation, physical activity, sleep.

\section{article details}

Article statistics: Word count: 3,661; Tables: 0; Figures: 0; References: 32

Received: March 2020; Accepted: April 2020; Published: June 2020

Full-text PDF: http://www.balticsportscience.com

Copyright @ Gdansk University of Physical Education and Sport, Poland

Indexation: Celdes, Clarivate Analytics Emerging Sources Citation Index (ESCI), CNKI Scholar (China National Knowledge Infrastructure), CNPIEC, De Gruyter - IBR (International Bibliography of Reviews of Scholarly Literature in the Humanities and Social Sciences), De Gruyter - IBZ (International Bibliography of Periodical Literature in the Humanities and Social Sciences), DOAJ, EBSCO - Central \& Eastern European Academic Source, EBSCO - SPORTDiscus, EBSCO Discovery Service, Google Scholar, Index Copernicus, J-Gate, Naviga (Softweco, Primo Central (ExLibris), ProQuest - Family Health, ProQuest - Health \& Medical Complete, ProQuest - Illustrata: Health Sciences, ProQuest - Nursing \& Allied Health Source, Summon (Serials Solutions/ProQuest, TDOne (TDNet), Ulrich's Periodicals Directory/ulrichsweb, WorldCat (OCLC)

Funding: This research received no specific grant from any funding agency in the public, commercial, or not-for-profit sectors.

Conflict of interests: Corresponding author:

Open Access License:
Authors have declared that no competing interest exists.

Aleksandra Jaroń; Department of Oral Surgery, Pomeranian Medical University in Szczecin; Powstańców Wielkopolskich 72/18, 70-111 Szczecin; e-mail: jaronola@gmail.com

This is an open access article distributed under the terms of the Creative Commons Attribution-Non-commercial 4.0 International (http://creativecommons.org/licenses/by-nc/4.0/), which permits use, distribution, and reproduction in any medium, provided the original work is properly cited, the use is non-commercial and is otherwise in compliance with the license. 


\section{INTRODUCTION}

With age, many people are affected and have symptoms of mild cognitive impairment. Over time, however, the likelihood of dementia significantly increases. In some cases, we recognize vascular dementia that affects small vessels in the brain. This may often be accompanied by small infarctions and brain tissue damage. The risk factors for Alzheimer's disease and other forms of dementia have a genetic component, which we have to bear in mind, but more often it is environmental factors (which we are able to control and change to a greater degree) that are associated with risk, as they, for example, exert vascular effects [1]. Research shows the impact and importance of selected nutrients as playing a special role in the development of Alzheimer's disease (AD). This is due to the fact that they are substrates for the synthesis of neurotransmitters in the Central Nervous System (CNS), and besides, they affect gene expression. This influence mainly results from epigenetic effects. However, they also function as antioxidants, preventing inflammation of the nervous system and, consequently, preventing $\mathrm{AD}[2]$.

Our particular focus is on risk factors such as smoking, social activity, physical activity and choosing the right diet - we focus our attention on them, because they are modifiable. It has already been proven that achieving apparent slowdown and reduction in cognitive decline is achieved by having a healthy diet. It is mainly done by way of limiting the consumption of sugars while maximizing the consumption of fish, vegetables, fruit, seeds and nuts. At the same time, it should be noted that studies have shown unfavourable correlations between cognitive functions and nutrients that increase the risk - these include trans fats as well as sugars. This may suggest that, for the most part, the same nutrients that contribute to a higher risk of cardiovascular disease also increase a risk of cognitive decline, or at least partially, by damaging the blood vessels in the brain. Particularly noteworthy in this matter is the Mediterranean diet as a lifestyle choice, thanks to which we may slow down and protect against cognitive decline in ageing patients [1]. Many studies have shown that the more you eat a western or highly processed diet, the more you are at risk for developing psychological symptoms, such as depression and anxiety. However, the more often we choose a Mediterranean-like diet, the better it protects us against the development of mental disorders [3]. Correlations between turmeric consumption and the development of $\mathrm{AD}$ were also studied [4].

In addition to diet, physical activity is also important. The benefits of physical activity are recognized as a great prophylactic option in both preventing cardiovascular diseases and diseases associated with ageing. However, physical activity is still not recommended enough and used insufficiently [5]. The Swedish National Board of Health and Welfare recommends that the healthcare system offer counselling combined with prescription exercises or a pedometer for people with insufficient physical activity [6]. Inactivity has a significant negative impact on health throughout life. However, other lifestyle factors, such as nutrition, smoking and excessive alcohol consumption, also disturb physiological functions during ageing [7].

A question arises: can supplementation also help? The treatment of mental illnesses, including major depressive disorder (MDD), is currently dominated by pharmacotherapy using antidepressants, as well as psychotherapy. However, these treatments help less than half of the patients, which suggests that additional strategies are needed to provide prevention and treatment 
for them. There are more and more studies suggesting that diet quality can lower the risk of developing a mental illness and affect mood. In addition, nutraceuticals, including n-3 fatty acids, folic acid, S-adenosylmethionine, vitamin $\mathrm{D}, \mathrm{N}$-acetylcysteine and probiotics are now promising and, therefore, widely studied. Bearing in mind the fact that patients stick to the nutraceutical approach better than to the traditional psychopharmacological treatment, the former seems to be the right choice [8].

If we combine what we know about our evolutionary heritage with the overwhelming recent evidence of the effects of physical inactivity, one clear conclusion emerges: being physically active is physiologically necessary to maintain health throughout our lives. Prospective and retrospective studies have shown that a lower incidence of cognitive impairment, depression, and dementia is found in people who maintain regular physical activity. Other animal and human studies show that exercise may change neuronal mechanisms, plasticity related to learning and memory, and can prevent agerelated cognitive decline and brain atrophy [7].

Therefore, this publication analyses the latest literature reports on the association of good health and well-being, including mental health, with modifiable factors, which we have an impact on in the context of research on various patient groups - in the context of diet, supplementation, exercise and the quality of sleep.

\section{MATERIAL AND METHODS}

The publications used in this work were sourced from the PubMed database and from Google Scholar. We searched for associations of modifiable factors affecting well-being and mental health. The index entries used to search the PubMed and Google Scholar browsers included diet, physical activity, supplementation, sleep and good functioning as well as mental health. The work uses articles published in English which are not older than 5 years.

\section{DIET}

As mentioned in the introduction, dietary ingredients may affect cognitive performance. Foods with a low glycaemic index seem to improve functional ability, memory and attention, while foods that are rich in monosaccharides cause difficulty in concentration. The brain needs a continuous supply of amino acids for the synthesis of neurotransmitters, especially catecholamines and serotonin. Low learning ability, worse memory and a reduced level of reasoning are associated with low serotonin levels. The quality and type of consumed fat also affects our intellectual abilities. Adequate nutrition is necessary to optimize brain functions and prevent cognitive decline [3].

\section{Psychonutrition}

A high intake of saturated fats is associated with cognitive decline, but consumption of polyunsaturated fatty acids (DHA - docosahexaenoic acid) effectively prevents it from worsening. It is advisable to follow diets with an appropriate ratio (5:1) of omega-6: 3 fatty acids (Mediterranean diet), given that they are associated with better memory and a lower risk of cognitive decline. Vitamins B1, B6, B12, B9 (folic acid) and D, choline, iron and iodine exert a neuroprotective effect and improve intellectual performance. Concurrently, antioxidants (vitamins C, E, A, zinc, selenium, lutein and zeaxanthin) play 
a very important role in defence against oxidative stress associated with mental deterioration and cognitive improvement [3]. A large part of these ingredients is contained in fruits and vegetables. The consumption of fish, fruit, vegetables, nuts and seeds is also beneficial [1].

Diet and nutrition can be an effective preventive measure against numerous mental illnesses. Nutritional psychiatry is a new, rapidly growing field of nutrition and mental health and is one of the ways to prevent them. The consequence of a bad diet is also an increased risk of obesity - a very dangerous global disease. Alzheimer's disease and depression are diseases associated with obesity, which produces a theory that vascular disorders may play a role in the development of dementia and other psychological pathologies. As a result of these observations, Alzheimer's disease and other dementias are now considered to be potentially preventable diseases.

Dozens of studies show that there is a relationship between diet in adulthood and the subsequent risk of developing PD (Parkinson's disease). People with $\mathrm{PD}$ are at an increased risk of malnutrition because of their increased metabolic demand and pathophysiology of the disease. The risk of malnutrition is additionally complicated by anosmia, difficulty in swallowing, constipation, and drug-nutrient interactions. More and more evidence shows that the gastrointestinal tract is affected in the early stages of the disease, which creates therapeutic options for early intervention. Diet modification and supplementation may alleviate the symptoms of constipation, depression, insomnia, dystonia, and help prevent cognitive impairment [9]. That is why a balanced diet is so important in preventing many diseases. Numerous studies show that the ketogenic diet may be used as a complimentary therapy for many diseases, including cancer. A low-carbohydrate and high-fat ketogenic diet was developed 100 years ago, initially to reduce seizures. To date, it has been an effective non-pharmacological treatment option for hard-to-control childhood epilepsy, although it is an underestimated form of treatment by many doctors. More recent evidence has demonstrated its effectiveness in treating motor and mental disorders and maintaining health in general [10].

However, the relationship between diet and mental health is a two-way process. The right proportions of macro- and micronutrients can positively affect our well-being; however, weakened mental health can contribute to an inability to buy and prepare healthy food. Instead, sweetened, processed products, that can briefly improve the mood, are picked [11].

\section{Low-carb diet}

The anti-tumour effect of KD in combination with low-dose chemotherapy (or without it) on neuroblastoma was studied. It was found that an increase in xenografts of neuroblastoma was significantly reduced at KD consisting of a ratio of 2:1 fat to carbohydrate + protein in combination with calorie restriction. It was found that ad libitum KD (8:1) with a fat content of $25 \%$ medium chain triglyceride and $75 \%$ long chain triglycerides gave a stronger anti-cancer effect compared to KD (8:1) with all long chain triglycerides, and was as effective as KD (2:1) in conjunction with calorie restriction, which was described above. These results underline the importance of optimized KD to counteract tumour growth and to sensitize it to chemotherapy without having to reduce calories [12]. Furthermore, it has been reported that KD may be potentially helpful in mild cognitive impairment, Parkinson's disease, schizophrenia, bipolar disorder and autism spectrum disorder [13]. 
Studies on the safety, tolerability, and intervention effectiveness of a very low carbohydrate diet (VLCK) compared to a low calorie diet in people with type II diabetes have shown that weight loss, reduced waist circumference, a decrease in HbA1c, and glycaemic control in the VLCK diet group were significantly greater in people using VLCK than in the control sample. No serious adverse events were reported, and mild AE in the VLCK diet group decreased during the last observation [14].

In addition, research is being conducted into the effects of the ketogenic diet on sleep quality, the thyroid function, the cardiovascular system and the cognitive function [15]. Studies performed by a research team from UK [16] on the effect of consuming wild blueberries (randomized double-blind study, placebo-controlled, for four weeks) in healthy 12-17-year-olds showed that daily consumption of wild blueberries (about $253 \mathrm{mg}$ of anthocyanins) significantly reduces symptoms of depression. However, no effect on anxiety was found. Wild blueberry supplementation may be a potential prevention strategy for depression in adolescents and may benefit mental health of the society [16].

Many dietary components may indirectly affect the genomic pathways that methylate DNA, and therefore gene expression. There is evidence of a biochemical relationship between nutrition quality and mental health. Deficiency in both macro- and microelements is associated with increased behavioural problems, and dietary supplementation has been found to be effective in treating some neuropsychiatric disorders [17].

\section{SUPPLEMENTATION}

\section{Microbiome}

A new path in nutritional psychiatry is to deepen research focused on modulation of the intestinal microflora (through probiotic and prebiotic foods and supplements) as a new therapy in the treatment of various neuropsychiatric diseases. A bidirectional communication between the intestinal microflora and the brain affects neurotransmission as well as behaviour often associated with neuropsychiatric conditions, and microbiome is associated with many systemic pathologies and obesity [18]. Recently, it has also been found that physical activity changes the intestinal microbiome [19].

Studies into probiotics have also provided evidence that taking probiotics by patients with mental disorders has a beneficial effect on HAMD (Hamilton Depression Rating Scale), CRP, IL-10 and MDA (malonaldehyde) levels [20].

Recent work has identified possible research goals exploring issues related to systemic and central vascular function, inflammation, metabolism, central activation, improvement of nerve performance and angiogenesis. The growing number of confirmed nutraceutical effects means that they are used more and more often and prescribed in the practice of psychiatrists [18].

\section{Lipids and neuropsychiatric diseases}

Studies have been conducted on the relationship of various membrane lipids to neuropsychic diseases, especially in schizophrenia spectrum disorders, and more recently in the early stages of psychosis. Seventy percent of the brain consists of lipids from various molecular classes and species. Most brain lipids are composed of diacyl classes enriched with polyunsaturated fatty acids 
(PUFAs) which are dominated by omega-3 and omega-6 molecules. The balance between omega- 3 and omega- 6 is important for neurological development. PUFAs are also involved in neurogenesis and neurotransmission. In rat models, omega-3 deficiency causes abnormalities in dopaminergic neurotransmission, affects the functioning of some receptors (including CB1 cannabinoids, $\mathrm{N}$-methyl-D-aspartate glutamate receptor, NMDA) and increases sensitivity to hallucinogens. However, omega-3 supplementation improves cognitive functions and prevents psychotic-like behaviours in some animal models of schizophrenia. It also reduces oxidative stress and prevents demyelination. In patients with mental disorders, a low omega- 3 ratio is more common than in the general population, but this phenomenon was not observed in all patients. This lipid abnormality may lead to myelination abnormalities and cognitive deficits observed in patients. It may also participate in oxidative stress disorders and inflammation reported in schizophrenia. On the other hand, the low omega-3 index deficit was associated with an increased cardiovascular risk, and omega-3 supplementation may also have a positive cardiovascular effect in psychiatric patients, even more so than in the general population. The presence of membrane lipid abnormalities also occurs in patients during the first psychotic episode (FEP). Omega-3 supplementation improved the recovery index and prevented grey matter loss in FEP. In patients with a very high risk of developing psychotic disorders (UHR), omega-3 supplementation is associated with a reduced risk of developing the disease. Despite the progress, the function and effect of membrane lipids are still poorly understood in schizophrenia. Omega-3 supplementation may be a promising treatment because of its good tolerability and acceptance by patients [21].

\section{Cancer diet}

Cancer treatment, such as chemotherapy, was an important part of prolonging survival in women diagnosed with breast cancer. However, chemotherapy may result in potentially toxic brain side effects that worsen memory, verbal fluidity and processing speed in up to $30 \%$ of women treated with it. Women report that cognitive deficits after chemotherapy negatively affect quality of life and may last up to ten years after treatment. The effect of omega-3 fatty acids and added sugars on cognitive function, neurological inflammation and hippocampal neurogenesis in adults was studied. In rodent models, omega-3 fatty acids co-administered with doxorubicin chemotherapy have been shown to prevent depressive behaviour and reduce neuritis, oxidative stress and neural apoptosis. In contrast, sugar-rich diets can interact with FA $\mathrm{n}-3$ to reduce their anti-inflammatory effects or act independently to increase neuritis, reduce hippocampal neurogenesis in adults, and promote cognitive deficits. It is best if a diet rich in long-chain, derived from marine omega-3 fatty acids and low in added sugars is an ideal formula for preventing or alleviating inflammation and oxidative stress, thus protecting neurons from the toxic effects of chemotherapy [22].

The effect of supplemented n-3 fatty acids on psychomotor and visual functions of mothers, premature babies and new-born babies was investigated. The results show that n-3 PUFA supplementation improves psychomotor and visual development in children, with no significant effect on the global IQ in later childhood [23].

Iodine and diet

Iodine deficiency (ID) in women of childbearing potential remains a global public health problem, mainly through the impact on foetal and infant 
neurological development. While iodine status is improving globally, ID is still common in pregnancy in which iodine demand is increased. Supplementation during pregnancy is recommended in some countries and supported by WHO when mandatory salt iodization is absent. Dairy products and seafood are the richest sources of iodine, and their consumption is necessary to ensure the proper level of iodine. Increased iodine through the diet may be possible if iodine-rich foods change their position in the diet because they currently only contribute to about $13 \%$ of the average energy intake of adult women. Iodine source products are sea products, fish, seafood, seaweed, dairy products and iodized salt [24].

Folic acid and vitamin $B 12$

Folic acid and vitamin B12 are well known as essential nutrients that play a key role in normal brain functions. Inflammatory processes play a role in the pathology of AD (Alzheimer's disease). Particular attention has been paid to effective nutritional intervention methods that reduce peripheral inflammatory cytokine levels to improve cognitive deficits. In a study of the effects of vitamin B12, folic acid and a combination of both, the folic acid group and vitamin B12 increased serum levels of homocysteine, vitamin B12, IL-6, and TNF- $\alpha$. This combination significantly changed the full-scale IQ, verbal IQ, information and results from Digit Span. The application of such therapy in the elderly with MCI for six months may significantly improve cognitive performance and reduce the level of inflammatory cytokines in human peripheral blood. The combination of folic acid and vitamin B12 was much better than folic acid alone or vitamin B12 alone [25].

\section{PHYSICAL ACTIVITY}

The recommendations for physical activity were prepared by Professional Associations for Physical Activity and approved by The Swedish Society of Medicine in 2011. All adults are recommended at least 150 minutes of moderate or 75 minutes of intensive aerobic exercise per week. More health benefits are achieved if those numbers are higher. The easiest and safest way of physical activity is walking (at least 10,000 steps a day or 6,000 steps a day in addition to daily activities). The elderly are also recommended balance exercises $[6,19]$. Physical activity, if properly performed, is an inexpensive and universal medicine with minimal side effects. This is our "home pharmacy" that we always have with us [19].

Patients at risk for atherosclerosis or any symptom of atherosclerosis (patients with coronary artery disease, stroke, peripheral artery disease) benefit from exercise, including patients with chronic heart failure. Physical activity also helps patients with lung diseases (COPD, asthma), metabolic diseases (diabetes, obesity, osteoporosis), as well as rheumatological diseases. Regular exercise improves cognitive functions, reduces depression and anxiety, and helps addicts, for example, to get out of addiction in the first stage of abstinence [19].

In the literature, we see studies on the impact of prenatal life in the context of a pregnant mother's lifestyle. Studies have shown that some unhealthy behaviours during pregnancy, such as sedentary lifestyles, may negatively affect the development of foetuses and children. The latest recommendations of the American College of Obstetrics and Gynecology (ACOG) for normally pregnant women indicate that they should exercise at least 30 minutes of moderate intensity physical activity on most days of the week. Physical activity 
during pregnancy may have a positive effect on new-borns and mothers this includes parameters such as gestational age, Apgar score, gestational diabetes, maternal weight gain and type of delivery 4-6. In addition, physical activity is associated with a reduction in the incidence and better management of postpartum depression. Correlation was also observed with better results in offspring in cognitive functions, academic performance and classroom behaviour [26].

Physical activity appears to be an effective complimentary therapy to reduce the severity of post-traumatic stress disorder (PTSD). Traditional treatment includes psychotherapy and pharmacotherapy. However, many people with this syndrome have limited access to these treatments. Physical activity has been shown to reduce post-traumatic stress disorder symptoms in people with sub-syndrome and those who are refractory to standard therapy. Physical activity has also been found to reduce the symptoms of other conditions that may be associated with PTSD (e.g. anxiety, depression, sleep disorders and cardiovascular diseases) [27].

Physical activity (PA) may be therapeutic for people with severe mental illness (SMI) who generally have low PA and experience numerous lifestyle-related medical complications. In the case of major depressive disorder (MDD), evidence clearly indicates that PA may improve depressive symptoms compared to the control group, with effects comparable to those provided by antidepressants and psychotherapy. PA may also improve cardiopulmonary fitness and quality of life for people with MDD. In the case of schizophrenia spectrum disorders, evidence suggests that aerobic PA may reduce psychiatric symptoms, improve cognition and various subdomains as well as cardio-respiratory performance [28].

The benefits of physical activity outweigh the risks [6]. Physical effort, if properly performed, is an inexpensive and universal medicine with minimal side effects. This is our "home pharmacy" that we always have with us [19].

\section{SLEEP}

Sleep plays a key role in the growth, development and health of a young body. The National Sleep Foundation recommends that young people aged 14-17 sleep 8-10 hours a day [29]. In their recent meta-analysis Olds et al. [30] found that the average night time in bed has decreased by 13.6 and 14.4 minutes with each year of age for boys and girls respectively, and that teenagers in the US sleep less than in Europe and Australia. In addition, some studies show that sleep time amongst American teens has decreased in a decade. Insufficient sleep, which is common in adolescents, was associated with adverse consequences, including mood disorders, other mental and physical illnesses, increased risk of injury, and poor school performance [31].

Sleep disorders and dementia are two common and significant health problems in the elderly. Studies show that sleep disorders may increase the risk of dementia. Numerous studies have also been conducted to predict the role of general sleep disorders on dementia related and unrelated to Alzheimer's disease and vascular dementia subtypes. Compared to people without sleep disorders, people who reported sleep disorders had a higher risk of dementia caused by any reason, AD and vascular dementia. Subgroup analysis showed that insomnia increases the risk of $\mathrm{AD}$, but not vascular dementia or resulting from any other reason [32]. 


\section{CONCLUSIONS}

Analysis of the latest literature has shown that there is evidence that modifiable factors have a positive effect on our well-being and health. The best effect can be achieved by combining positive actions in the field of diet, supplementation, moderate but systematic physical activity, and adding the right amount of sleep per day. These are low-cost activities available to every patient. There are definitely no contraindications to use them to improve your functioning and well-being.

\section{REFERENCES}

[1] Tucker KL. Nutrient intake, nutritional status, and cognitive function with aging. Ann N Y Acad Sci. 2016;1367(1):38-49. https://doi.org/10.1111/nyas.13062

[2] Muñoz Fernández SS, Lima Ribeiro SM. Nutrition and Alzheimer Disease. Clin Geriatr Med. 2018;34(4):677-697. https://doi.org/10.1016/j.cger.2018.06.012

[3] Martínez García RM, Jiménez Ortega AI, López-Sobaler AM, Ortega RM. Estrategias nutricionales que mejoran la función cognitiva. Nutr Hosp 2018;35(6):16-19. https://doi.org/10.20960/nh.2281

[4] Botchway BOA, Moore MK, Akinleye FO, Iyer IC, Fang M. Nutrition: Review on the possible treatment for Alzheimer's Disease. J Alzheimers Dis. 2018;61(3):867-883. https://doi.org/10.3233/JAD-170874

[5] Orkaby AR, Forman DE. Physical activity and CVD in older adults: an expert's perspective. Expert Rev Cardiovasc Ther. 2018;16(1):1-10. https://doi.org/10.1080/14779072.2018.1419062

[6] Jansson E, Hagströmer M, Anderssen SA. Physical activity--new paths and choices in the recommendations for adults. Lakartidningen. 2015;17:112.

[7] Harridge SD, Lazarus NR. Physical Activity, Aging, and Physiological Function. Physiology (Bethesda). 2017;322:152-161. https://doi.org/10.1152/physiol.00029.2016

[8] di Michele F, Talamo A, Niolu C, Siracusano A. Vitamin D and N-Acetyl cysteine supplementation in treatment resistant depressive disorder patients: A general review. Curr Pharm Des. 2020 Apr 5. https://doi.org/10.2174/1381612826666200406090051

[9] Mischley LK. Nutrition and Nonmotor Symptoms of Parkinson's Disease. Int Rev Neurobiol. 2017;134:1143-1161. https://doi.org/10.1016/bs.irn.2017.04.013

[10] Barry D, Ellul S, Watters L, Lee D, Haluska R Jr, White R. The ketogenic diet in disease and development. Int J Dev Neurosci. 2018;68:53-58. https://doi.org/10.1016/j.ijdevneu.2018.04.005

[11] an der Pols JC. Nutrition and mental health: bidirectional associations and multidimensional measures. Public Health Nutr. 2018;21(5):829-830. https://doi.org/10.1017/S1368980017003974

[12] Weber DD, Aminazdeh-Gohari S, Kofler B. Ketogenic diet in cancer therapy. Aging (Albany NY). 2018;10(2):164-165. https://doi.org/10.18632/aging.101382

[13] Morris G, Maes M, Berk M, Carvalho AF, Puri BK. Nutritional ketosis as an intervention to relieve astrogliosis: Possible therapeutic applications in the treatment of neurodegenerative and neuroprogressive disorders. Eur Psychiatry. 202063(1):e8

[14] Goday A, Bellido D, Sajoux I, Crujeiras AB, Burguera B, García-Luna PP, Oleaga A, Moreno B, Casanueva FF. Short-term safety, tolerability and efficacy of a very low-calorie-ketogenic diet interventional weight loss program versus hypocaloric diet in patients with type 2 diabetes mellitus. Nutr Diabetes. 2016;6(9):e230. https://doi.org/10.1038/nutd.2016.36

[15] Iacovides S, Meiring RM. The effect of a ketogenic diet versus a high-carbohydrate, low-fat diet on sleep, cognition, thyroid function, and cardiovascular health independent of weight loss: study protocol for a randomized controlled trial. Trials. 2018;19(1):62. https://doi.org/10.1186/s13063-018-2462-5

[16] Fisk J, Khalid S, Reynolds SA, Williams CM. Effect of 4 weeks daily wild blueberry supplementation on symptoms of depression in adolescents. Br J Nutr.2020;10:1-8. https://doi.org/10.1017/ S0007114520000926

[17] Stevens AJ, Rucklidge JJ, Kennedy MA. Epigenetics, nutrition and mental health. Is there a relationship? Nutr Neurosci. 2018;21(9):602-613. https://doi.org/10.1080/1028415X.2017.1331524

[18] Owen L, Corfe B. The role of diet and nutrition on mental health and wellbeing. Proc Nutr Soc. 2017;76(4):425-426. https://doi.org/10.1017/S0029665117001057

[19] Tuka V, Daňková M, Riegel K, Matoulek M. Physical activity - the Holy Grail of modern medicine?. Vnitr Lek. Fall 2017;63(10):729-736.

[20] Amirani E, Milajerdi A, Mirzaei H, Jamilian H, Mansournia MA, Hallajzadeh J,Ghaderi A. The effects of probiotic supplementation on mental health, biomarkers of inflammation and oxidative stress in patients with psychiatric disorders: A systematic review and meta-analysis of randomized controlled trials. Complement Ther Med. 2020;49:102361. https://doi.org/10.1016/j.ctim.2020.102361

[21] Frajerman A, Kebir O, Chaumette B, Tessier C, Lamazière A, Nuss P, Krebs MO. Membrane lipids in schizophrenia and early phases of psychosis: Potential biomarkers and therapeutic targets?. Encephale. 2020.

[22] Orchard TS, Gaudier-Diaz MM, Weinhold KR, Courtney DeVries A. Clearing the fog: a review of the effects of dietary omega-3 fatty acids and added sugars on chemotherapy-induced cognitive deficits. Breast Cancer Res Treat. 2017;161(3):391-398. https://doi.org/10.1007/s10549-016-4073-8 
[23] Shulkin M, Pimpin L, Bellinger D, et al. n-3 Fatty Acid supplementation in mothers, preterm infants, and term infants and childhood psychomotor and visual development: A systematic review and metaanalysis. J Nutr. 2018;148(3):409-418. https://doi.org/10.1093/jn/nxx031

[24] Bouga M, Lean MEJ, Combet E. Contemporary challenges to iodine status and nutrition: The role of foods, dietary recommendations, fortification and supplementation. Proc Nutr Soc. 2018;77(3):302313. https://doi.org/10.1017/S0029665118000137

[25] Ma F, Zhou X, Li Q, et al. Effects of folic acid and vitamin b12, alone and in combination on cognitive function and inflammatory factors in the elderly with mild cognitive impairment: a single-blind experimental design. Curr Alzheimer Res. 2019;16(7):622-632. https://doi.org/10.2174/15672050 16666190725144629

[26] Álvarez-Bueno C, Cavero-Redondo I, Sánchez-López M, Garrido-Miguel M, Martínez-Hortelano JA, Martínez-Vizcaíno V. Pregnancy leisure physical activity and children's neurodevelopment: A narrative review. BJOG. 2018;125(10):1235-1242. https://doi.org/10.1111/1471-0528.15108

[27] Oppizzi LM, Umberger R. The effect of physical activity on PTSD. Issues Ment Health Nurs. 2018;39(2):179-187. https://doi.org/10.1080/01612840.2017.1391903

[28] Stubbs B, Vancampfort D, Hallgren M, et al. EPA guidance on physical activity as a treatment for severe mental illness: a meta-review of the evidence and Position Statement from the European Psychiatric Association (EPA), supported by the International Organization of Physical Therapists in Mental Health(IOPTMH). Eur Psychiatry. 2018;54:124-144. https://doi.org/10.1016/j.eurpsy.2018.07.004

[29] Hirshkowitz M, Whiton K, Albert SM, Ware J. National Sleep Foundation's updated sleep duration recommendations: final report. Sleep Health 2016;2:233-43. https://doi.org/10.1016/j.sleh.2015.10.004

[30] Olds T, Blunden S, Petkov J, Forchino F. The relationships between sex, age, geography and time in bed in adolescents: a meta-analysis of data from 23 countries. Sleep Med Rev. 2010;14:371-337. https://doi.org/10.1016/j.smrv.2009.12.002

[31] Zhang J, Paksarian D, Lamers F, Hickie IB, He J, Merikangas KR. Sleep Patterns and Mental Health Correlates in US Adolescents. J Pediatr. 2017;182:137-143. https://doi.org/10.1016/j.jpeds.2016.11.007

[32] Shi L, Chen SJ, Ma MY, Bao YP, Han Y, Wang YM, Shi J, Vitiello MV, Lu L. Sleep disturbances increase the risk of dementia: A systematic review and meta-analysis. Sleep Med Rev. 2018;40:4-16. https:// doi.org/10.1016/j.smrv.2017.06.010 